\title{
A NONIDENTITY FOR RIGHT ALTERNATIVE RINGS
}

\author{
ERWIN KLEINFELD AND MARGARET HUMM KLEINFELD ${ }^{1}$
}

Previously we established that the free alternative ring on four or more generators had a specific element whose cube is zero [1]. A ring is defined to be right alternative in case its elements satisfy the identity

$$
(x, y, y)=0 \text { and }(x, y, z)=-(x, z, y),
$$

and strongly right alternative if in addition its elements also satisfy the identity

$$
(x y \cdot z) y=x(y z \cdot y) .
$$

In rings of characteristic not two, (1) implies (2) [2], while in rings of characteristic two this is not the case [3].

In the following note we establish that in the free strongly right alternative ring $R$ generated by $a$ and $b$ we have $((a, b), a, b) \neq 0$. We also know from previous work [2, Lemma 5(i)] that $((a, b), a, b)^{2}=0$ in $R$. While it was already known from theoretical considerations that the free right alternative ring of characteristic not two on two or more generators would have to have at least one element $x \neq 0$ such that $x^{2}=0$, since otherwise the Main Theorem of [2] implies it would have to be alternative, which we know is not the case, this enables one to locate such an element specifically for the first time.

In order to prove that $((a, b), a, b) \neq 0$ in $R$, it suffices to give an example of a strongly right alternative algebra in which this is not an identity. Our example has basis elements $q_{1}, \cdots, q_{9}$. The only nonzero products of basis elements are the following twelve: $q_{1}^{2}=q_{3}, q_{1} q_{2}$ $=q_{5}, q_{2} q_{1}=q_{6}, q_{2}^{2}=q_{4}, q_{2} q_{7}=q_{9}, q_{3} q_{2}=-q_{7}, q_{4} q_{1}=-q_{8}, q_{4} q_{3}=-q_{9}, \quad q_{5} q_{1}$ $=q_{7}, q_{6} q_{2}=q_{8}, q_{6} q_{5}=q_{9}$, and $q_{8} q_{1}=q_{9}$.

From this information one may construct the multiplication table for the basis elements of the algebra. To see that the algebra $A$ thus defined is right alternative, it is sufficient to verify (1) whenever $x, y$, and $z$ are basis elements. The identity must then hold for all elements because of the linearity of the associator.

It can easily be verified that $q_{\overline{7}}, q_{8}$, and $q_{9}$ are in the nucleus and so (1) is automatically satisfied if any of these appear. It can also be verified that $q_{i} R \cdot R=0=q_{i} R^{2}$ for $i=3,5$. Hence (1) holds if $x=q_{3}$ or

Received by the editors August 23, 1968.

1 This paper was supported in part by a grant from the U. S. Army Research Office (Durham). 
$q_{5}$. Now $q_{6} r \cdot s=0$ unless $r=q_{2}$ and $s=q_{1}$ while $q_{6} \cdot u v=0$ unless $u=q_{1}$ and $v=q_{2}$. Using the table we see that $\left(q_{6}, q_{1}, q_{2}\right)=-q_{9}$ and $\left(q_{6}, q_{2}, q_{1}\right)$ $=q_{9}$. Thus (1) holds for $x=q_{6}$. Similarly, $q_{4} r \cdot s=0$ unless $r=s=q_{1}$ and also $q_{4} \cdot u v=0$ unless $u=v=q_{1}$, but since $q_{4} q_{1} \cdot q_{1}=-q_{9}=q_{4} \cdot q_{1}^{2}$, (1) holds if $x=q_{4}$. If $x=q_{2}$, the nonzero associators are $\left(q_{2}, q_{1}, q_{2}\right)=q_{8},\left(q_{2}, q_{2}, q_{1}\right)$ $=-q_{8},\left(q_{2}, q_{1}, q_{5}\right)=q_{9},\left(q_{2}, q_{5}, q_{1}\right)=-q_{9},\left(q_{2}, q_{2}, q_{1}\right)=-q_{8},\left(q_{2}, q_{1}, q_{2}\right)=q_{8}$, $\left(q_{2}, q_{2}, q_{3}\right)=-q_{9}$, and $\left(q_{2}, q_{3}, q_{2}\right)=q_{9}$. Thus (1) is verified for $x=q_{2}$. If $x=q_{1}$, the nonzero associators are $\left(q_{1}, q_{1}, q_{2}\right)=-q_{7}$ and $\left(q_{1}, q_{2}, q_{1}\right)=q_{7}$ and all the rest vanish. Thus we have shown that (1) holds in all cases. To see that $A$ also satisfies (2) it is sufficient to check that

$$
(x y \cdot z) w+(x w \cdot z) y=x(y z \cdot w)+x(w z \cdot y)
$$

whenever $x, y, z$, and $w$ are all basis elements. From the table one finds that the only nonzero words of the form $(x y \cdot z) w$ are $\left(q_{2} q_{1} \cdot q_{2}\right) q_{1}$ and $\left(q_{2} q_{2} \cdot q_{1}\right) q_{1}$, while the only nonzero words of the form $x(y z \cdot w)$ are $q_{2}\left(q_{1} q_{1} \cdot q_{2}\right)$ and $q_{2}\left(q_{1} q_{2} \cdot q_{1}\right)$. Since $\left(q_{2} q_{1} \cdot q_{2}\right) q_{1}=q_{9}=q_{2}\left(q_{1} q_{2} \cdot q_{1}\right)$ and $\left(q_{2} q_{2} \cdot q_{1}\right) q_{1}=-q_{9}=q_{2}\left(q_{1} q_{1} \cdot q_{2}\right)$ we see that (3) must hold in $A$.

Having shown that $A$ is strongly right alternative, one may choose $a=q_{1}, b=q_{2}$. It is clear that $a$ and $b$ generate $A$ and we see that $((a, b), a, b)=\left(q_{5}-q_{6}, q_{1}, q_{2}\right)=\left(q_{5}, q_{1}, q_{2}\right)-\left(q_{6}, q_{1}, q_{2}\right)=-\left(q_{6}, q_{1}, q_{2}\right)$ $=q_{6} q_{5}=q_{9} \neq 0$.

\section{BIBLIOGRAPHY}

1. Margaret M. Humm and Erwin Kleinfeld, On free alternative rings, J. Combinatorial Theory 2 (1967), 140-144.

2. Erwin Kleinfeld, Right alternative rings, Proc. Amer. Math. Soc. 4 (1953), 939944.

3. R. L. San Soucie, Right alternative division rings of characteristic 2, Proc. Amer. Math. Soc. 6 (1955), 291-296.

Syracuse UNIVERSITY AND

UNIVERSITY OF IOWA 